\title{
CLOSE COMPANIONS TO TWO HIGH-REDSHIFT QUASARS*
}

\author{
Ian D. McGreer ${ }^{1}$, Xiaohui Fan ${ }^{1}$, Michael A. Strauss ${ }^{2}$, Zoltàn Haiman ${ }^{3}$, Gordon T. Richards ${ }^{4}$, \\ Linhua JiAnG $^{5,8}$, Fuyan Bian ${ }^{1,6}$, AND Donald P. SchneIder ${ }^{7}$ \\ ${ }^{1}$ Steward Observatory, The University of Arizona, 933 North Cherry Avenue, Tucson, AZ 85721-0065, USA; imcgreer@as.arizona.edu \\ ${ }^{2}$ Princeton University Observatory, Peyton Hall, Princeton, NJ 08544, USA \\ ${ }^{3}$ Department of Astronomy, Columbia University, 550 West 120th Street, New York, NY 10027, USA \\ ${ }^{4}$ Department of Physics, Drexel University, 3141 Chestnut Street, Philadelphia, PA 19104, USA \\ ${ }^{5}$ School of Earth and Space Exploration, Arizona State University, Tempe, AZ 85287, USA \\ ${ }^{6}$ Research School of Astronomy and Astrophysics, Australian National University, Canberra, ACT 2611, Australia \\ ${ }^{7}$ Department of Astronomy and Astrophysics and the Institute for Gravitation and the Cosmos, The Pennsylvania State University, University Park, PA 16802, USA \\ Received 2014 April 9; accepted 2014 July 12; published 2014 September 15
}

\begin{abstract}
We report the serendipitous discoveries of companion galaxies to two high-redshift quasars. SDSS J025617.7+001904 is a $z=4.79$ quasar included in our recent survey of faint quasars in the SDSS Stripe 82 region. The initial MMT slit spectroscopy shows excess Ly $\alpha$ emission extending well beyond the quasar's light profile. Further imaging and spectroscopy with LBT/MODS1 confirms the presence of a bright galaxy $\left(i_{\mathrm{AB}}=23.6\right)$ located $2^{\prime \prime}\left(12 \mathrm{kpc}\right.$ projected) from the quasar with strong Ly $\alpha$ emission $\left(\mathrm{EW}_{0} \approx 100 \AA\right)$ at the redshift of the quasar, as well as faint continuum. The second quasar, CFHQS J005006.6+344522 $(z=6.25)$, is included in our recent HST SNAP survey of $z \sim 6$ quasars searching for evidence of gravitational lensing. Deep imaging with ACS and WFC3 confirms an optical dropout $\sim 4.5 \mathrm{mag}$ fainter than the quasar $\left(Y_{\mathrm{AB}}=25\right)$ at a separation of $0 \prime 9$. The red $i_{775}-Y_{105}$ color of the galaxy and its proximity to the quasar (5 kpc projected if at the quasar redshift) strongly favor an association with the quasar. Although it is much fainter than the quasar, it is remarkably bright when compared to field galaxies at this redshift, while showing no evidence for lensing. Both systems may represent late-stage mergers of two massive galaxies, with the observed light for one dominated by powerful ongoing star formation and for the other by rapid black hole growth. Observations of close companions are rare; if major mergers are primarily responsible for high-redshift quasar fueling then the phase when progenitor galaxies can be observed as bright companions is relatively short.
\end{abstract}

Key words: galaxies: high-redshift - quasars: general - quasars: individual (SDSS J025617.7+001904, CFHQSJ005006.6+344522)

Online-only material: color figures

\section{INTRODUCTION}

The discovery of quasars as distant as $z \sim 7$ already powered by black holes (BHs) with masses $\sim 10^{9} M_{\odot}$ (Fan et al. 2001; Mortlock et al. 2011; Venemans et al. 2013) presents a challenge for early structure formation models. First, some physical process is needed to generate seed BHs at even higher redshifts (e.g., Volonteri 2010; Haiman 2013). Next, regardless of the nature of the seeds, the initial BHs must grow rapidly in order to reach a billion solar masses or more in less than a Gyr. $\Lambda \mathrm{CDM}$ models generally predict that this high-redshift growth occurs by gas accretion rather than by a succession of $\mathrm{BH}$ mergers $(\mathrm{Li}$ et al. 2007; Di Matteo et al. 2008; Tanaka \& Haiman 2009), requiring both a enormous fuel supply and a mechanism for driving the gas from intergalactic scales down to the central regions of galaxies where it can be accreted onto the $\mathrm{BH}$.

Mergers of gas-rich disk galaxies have been proposed as a solution to this problem (e.g., Kauffmann \& Haehnelt 2000; Hopkins et al. 2006; Li et al. 2007; Hopkins et al. 2008). In the merger scenario the remnant typically passes through an obscured starburst phase followed by a luminous, unobscured

\footnotetext{
* Based in part on observations made with the NASA/ESA Hubble Space Telescope, obtained at the Space Telescope Science Institute, which is operated by the Association of Universities for Research in Astronomy, Inc., under NASA contract NAS 5-26555. These observations are associated with programs \#12184 and \#12493. Observations were also made with the LBT and MMT.

8 Hubble Fellow.
}

quasar phase. Merger dynamics provide a natural mechanism for shedding angular momentum and driving gas to central regions (Hernquist 1989), plausibly accounting for the continuous fuel supply required to grow high-redshift quasars.

While the merger hypothesis is consistent with many observations, direct evidence for merger activity associated with individual quasars is rare. This is likely because the observational signatures of a recent merger (tidal tails, gas shells, etc.; see, e.g., Bennert et al. 2008) are short-lived and faint, thus easily overwhelmed by the luminous quasar. Nonetheless, early Hubble Space Telescope (HST) observations showed that at least some nearby luminous quasars show evidence for recent interactions, with a significant fraction having close $(<10 \mathrm{kpc})$ companion galaxies (Bahcall et al. 1997). In addition, the fraction of highly dust-obscured quasars showing evidence of recent merger activity is close to unity (Urrutia et al. 2008), in agreement with the general outline of the merger scenario.

Mergers are more likely to occur in overdense environments where the rate of galactic encounters is enhanced. Even if direct evidence for recent merging activity in quasar host galaxies is elusive, circumstantial evidence in the form of local overdensities may argue in favor of merger-driven growth. For example, in the merger-tree-based hydrodynamical simulation of Li et al. (2007), a single $z \sim 6.5$ quasar is assembled from a succession of seven major mergers, so that the quasar is surrounded by nearby $(\lesssim 20 \mathrm{kpc})$ companions more or less continuously from $z \sim 12$ to $z \sim 7$ (see Figure 6 of Li et al. 2007). More broadly, the expectation that exceedingly rare 

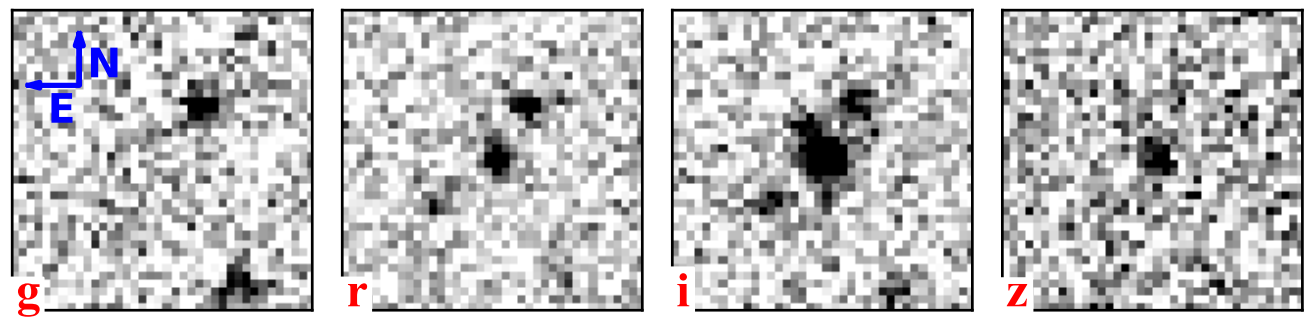

Figure 1. SDSS deep Stripe 82 griz images of J0256+0019. Images are $20^{\prime \prime}$ on a side with a pixel scale of 0.45 and are oriented with north up and east to the left. The companion galaxy is visible $2^{\prime \prime} \mathrm{NE}$ of the quasar in the $i$-band image. Two additional galaxies are detected to the NW and SE at distances of $\sim 5^{\prime \prime}$ from the quasar and are likely at low redshift given their relatively blue colors.

(A color version of this figure is available in the online journal.)

high-redshift quasars would occupy the most highly biased regions at their observed epoch has motivated many surveys of their environments on few Mpc scales (Stiavelli et al. 2005; Willott et al. 2005; Zheng et al. 2006; Kashikawa et al. 2007; Kim et al. 2009; Utsumi et al. 2010; Benítez et al. 2013; Husband et al. 2013), the results of which have been inconclusive, with some quasar fields having apparent overdensities of candidate associated galaxies, and some not. Some recent theoretical models even suggest that high-redshift quasars do not inhabit the strongest large-scale overdensities at high redshift (Fanidakis et al. 2013).

A particularly well-studied case of prodigious merger activity associated with a luminous, high-redshift quasar is BR 1202-0725 at $z=4.7$. One of the first detections of a FIR-hyperluminous, high-redshift submillimeter galaxy (SMG) resulted from observations of the host galaxy of this quasar (Isaak et al. 1994). Subsequently, an optically undetected SMG as bright as the quasar host at submillimeter wavelengths was discovered a mere $4^{\prime \prime}$ from the quasar (Omont et al. 1996), as was a pair of Ly $\alpha$-emitting galaxies within $\sim 2^{\prime \prime}$ ( $\mathrm{Hu}$ et al. 1996; Petitjean et al. 1996). BR 1202-0725 has been dubbed the "archetypal" system of a close group of galaxies leading to mergers and fueling both supermassive black hole (SMBH) formation and prodigious star formation (Carilli et al. 2013). It is curious, however, that observations of systems like BR 12020725 are rare, if indeed group-scale mergers are a key pathway to forming high-redshift quasars.

In this paper we report the discovery of close companions ( $\lesssim 10 \mathrm{kpc}$ ) of two high-redshift quasars. The first example is a companion galaxy to the $z \sim 5$ quasar SDSS J025617.7+001904 (hereafter J0256+0019). This galaxy is located $2^{\prime \prime}$ from the quasar and has strong $\operatorname{Ly} \alpha$ emission at the same redshift. It bears many similarities to the Ly $\alpha$-emitting companions of BR 1202-0725. We present imaging and spectroscopy of this system in Section 2. The second quasar, CFHQS J005006.6+344522 (hereafter J0050+3445), was included in an HST SNAP survey of $z \sim 6$ quasars searching for evidence of gravitational lensing. A galaxy 0.9 from the quasar was detected in the $H S T$ image. In Section 3 we present strong evidence from $H S T$ imaging that this galaxy is almost certainly associated with the quasar. In Section 4 we consider the physical origin of the observed emission from both galaxies, discuss these observations in the context of the merger hypothesis, and draw rough conclusions on the incidence of close companions for similar quasars. We present brief conclusions and speculate on the nature of companion galaxies in Section 5.

All magnitudes are on the AB system (Oke \& Gunn 1983) and have been corrected for Galactic extinction using the Schlegel et al. (1998) extinction maps unless otherwise noted. We adopt a
$\Lambda$ CDM cosmology with parameters $\Omega_{\Lambda}=0.727, \Omega_{m}=0.273$, $\Omega_{\mathrm{b}}=0.0456$, and $H_{0}=70 \mathrm{kms}^{-1} \mathrm{Mpc}^{-1}$ (Komatsu et al. 2011) when needed.

\section{J0256+0019}

The quasar J0256+0019 was selected as a $z \sim 5$ quasar candidate based on optical and near-IR color selection (McGreer et al. 2013). The input imaging included deep coadded ugriz (Fukugita et al. 1996) images from the Sloan Digital Sky Survey (SDSS; York et al. 2000) Stripe 82 region, with $\sim 80-100$ individual images contributing to the coadd at each position (see Jiang et al. 2009 and Jiang et al. 2014 for details). These images reach $\sim 2$ mag deeper than single-epoch SDSS imaging. $J$-band imaging from the UKIRT Infrared Deep Sky Survey (UKIDSS; Lawrence et al. 2007) was also used for the color selection. In this section we describe subsequent observations of the quasar and companion galaxy using MMT and Large Binocular Telescope (LBT).

\subsection{MMT Observations}

J0256+0019 was confirmed as a $z=4.8$ quasar using longslit spectroscopic observations obtained with the MMT Red Channel spectrograph on 2011 October 1. The Red Channel observations were performed with a $1^{\prime \prime} \times 180^{\prime \prime}$ slit and the low dispersion $270 \mathrm{~mm}^{-1}$ grating, delivering a resolution of $R \sim$ 640 over the range $5700 \AA$ to $9700 \AA$. Three 10 m exposures were combined to produce the final spectrum. Data were processed in a standard fashion; details are given in McGreer et al. (2013).

We immediately noticed extended Ly $\alpha$ emission in the twodimensional (2D) spectrum. Although the seeing during the spectroscopic observations was $\sim 0^{\prime \prime} .8$, the emission in the vicinity of the quasar's Ly $\alpha$ line extends to roughly $2^{\prime \prime}$. The Ly $\alpha$ detection was purely serendipitous: only after the observations did we notice extended emission in the same location in the stacked $i$-band image from Stripe 82 (Figure 1). Although we applied no morphological criteria in selecting quasar candidates from Stripe 82, J0256+0019 would have likely been targeted anyway, as the $i$-band detection for the quasar has SExtractor CLASS_STAR $=0.95$ (where 1 represents a stellar profile); the excess $i$-band emission is quite weak relative to the quasar.

We obtained near-IR imaging in the $J$ and $H$ bands with the MMT Smithsonian Widefield Infrared Camera (SWIRC; Brown et al. 2008) on 2011 October 15. The seeing was 0.7 and conditions were variable and non-photometric. J0256+0019 was observed in the $J$ and $H$ bands using dithered integrations for a total exposure time of $20.7 \mathrm{~m}$ and $16.3 \mathrm{~m}$, respectively. The images were shifted and stacked, and then registered to an astrometric solution obtained by matching UKIDSS stars 

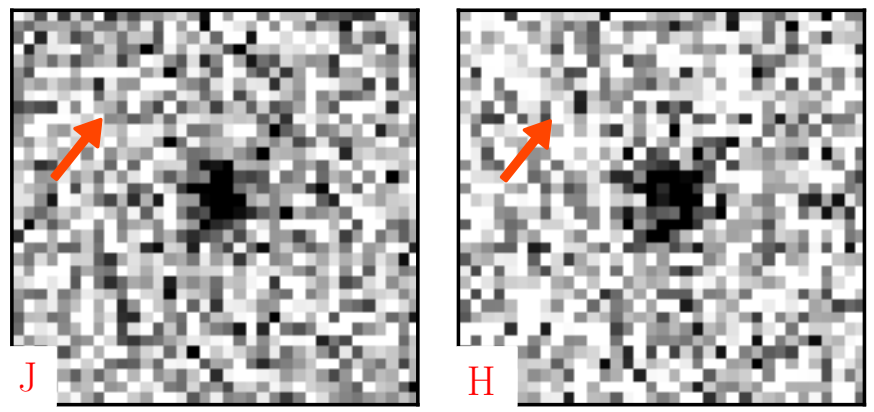

Figure 2. MMT SWIRC $J$ and $H$ images of J0256+0019. Images are $5^{\prime \prime}$ on a side with a pixel scale of 0 "' 15 and are oriented with north up and east to the left. The position of the companion galaxy is marked with an arrow. The quasar is detected in both images but the companion galaxy is not.

(A color version of this figure is available in the online journal.)

within the field. Flux calibration was obtained from observations of faint UKIRT standard stars from Leggett et al. (2006) and checked against UKIDSS matches. The quasar is clearly detected in both images, with fluxes of $J=22.11 \pm 0.14$ and $H=21.43 \pm 0.10$ measured from $1^{\prime \prime} .8$ apertures (Figure 2). The companion object is not detected to $3 \sigma$ limits of $J=22.8$ and $H=23.2$ (both $\mathrm{AB})$.

\subsection{LBT/MODS Observations}

Additional imaging and spectroscopic observations were obtained with the LBT Multi-Object Double Spectrograph (MODS1) instrument on 2012 September 21. MODS1 is an optical imager/spectrograph mounted on the LBT $2 \times 8.4 \mathrm{~m}$ telescope (Pogge et al. 2006). We obtained both direct imaging and grating spectroscopy of J0256+0019. During the observations conditions were non-photometric with mostly clear skies and good seeing, improving from $0 .{ }^{\prime \prime} 8$ to 0.6 .

\subsubsection{Imaging}

In acquisition mode the MODS 1 red CCD employs a $1024 \times 1024$ array with a pixel scale of $0{ }^{\prime} .123$ and a FOV of 2 ". 1 . Immediately following a series of longslit spectroscopic observations (see below), a single $600 \mathrm{~s}$ exposure was obtained with the SDSS $i$ filter. Guiding was continuous throughout both observations.

The $i$-band image was reduced in a standard fashion. A bias correction was applied from a median of bias images. Sky flats were obtained at twilight and used to provide the flat field. The image was masked of regions affected by scattered light from a nearby bright star and from obstruction by the guide probe camera, leaving roughly $2 / 3$ of the field usable. Astrometric registration and flux calibration were obtained by matching stellar objects from the Stripe 82 coadded imaging. Object detection was performed with SExtractor (Bertin \& Arnouts 1996) using standard parameters.

We measure a separation between the quasar and the companion object of 1 1.8 from the MODS1 $i$-band image, corresponding to a projected physical separation of $12.1 \mathrm{kpc}$ for our adopted cosmology. The quasar has $i=22.08 \pm 0.02$, somewhat fainter than measured from the Stripe 82 coadded imaging $(i=21.73 \pm 0.02)$. This is likely due to blending, although variability could also contribute to the flux difference. The two objects are well resolved in the MODS1 image (Figure 3). According to the SExtractor star/galaxy separation, the quasar is consistent with a point source (CLASS_STAR $=0.97$ ) while the

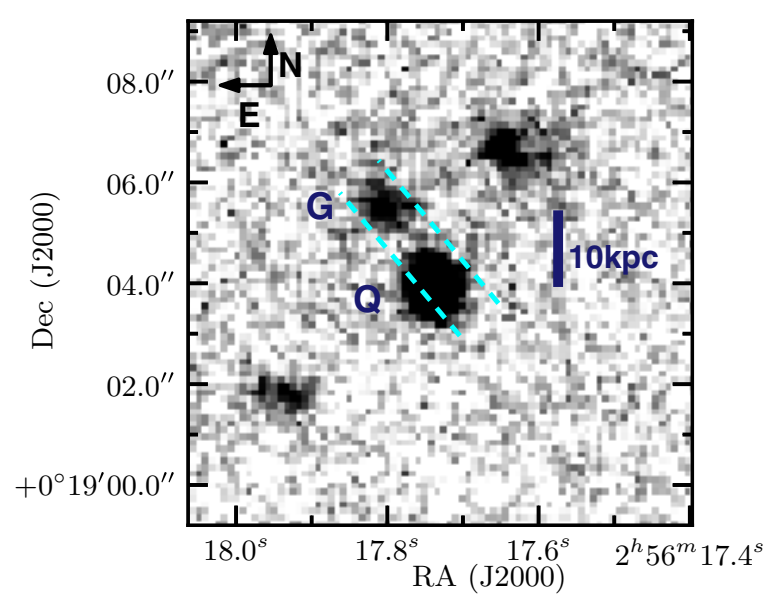

Figure 3. LBT/MODS $i$-band image of J0256+0019. The scale is $0^{\prime \prime} 123 \mathrm{pixel}^{-1}$ and the seeing is $\sim 00^{\prime \prime} 6$. The companion galaxy (labeled $\mathrm{G}$ ) is easily resolved from the quasar (Q) and is extended compared to stars within the field. The galaxy has $i=23.6$. The dashed cyan lines correspond to the orientation of the slit during the spectroscopic observations.

(A color version of this figure is available in the online journal.)

companion is extended (CLASS_STAR $=0.17$ ). The companion has $i=23.60 \pm 0.07$ and measured size of FWHM $=1^{\prime \prime}$.0 (compared to 0'.57 for the quasar). The companion is not detected in the Stripe 82 coadded $z$-band image to a $3 \sigma$ limit of $z=23.5$; so $i-z \lesssim 0.1$ for this object.

\subsubsection{Spectroscopy}

In grating spectroscopic mode the MODS1 Red channel utilizes a $8 \mathrm{k} \times 3 \mathrm{k}$ array. We used the single grating mode with the G670L grating and a $1^{\prime \prime}$ longslit, providing a spectral resolution of $R \sim 1400$ and wavelength coverage from $5800 \AA$ to $1 \mu \mathrm{m}$ at a dispersion of $0.8 \AA \mathrm{pixel}^{-1}$. Three exposures of 1200 s each were obtained.

The position angle between the quasar and the companion is 31.2 from the MODS 1 imaging. We estimated a P.A. of $40^{\circ}$ from the Stripe 82 coadded imaging and placed the MODS longslit at this angle for the spectroscopic observations. Although the slit angle was offset by $\sim 9^{\circ}$ from the true P.A. between the two objects, the quasar was also slightly off-center relative to the slit, while the companion galaxy was relatively well-centered.

The images were first processed with the MODS CCD reduction utilities (modsTools v0.3) to obtain bias and flat-field corrections. Transformations between image pixels and wavelength were determined from polynomial fits to arc calibration images. A 2D sky model was fit to each image using b-splines (Kelson 2003) and then subtracted. Cosmic rays were identified and masked during the construction of the sky model. The individual exposures were combined with inverse variance weighting to produce the final 2D spectrum.

Figure 4 displays a section of the sky-subtracted and combined 2D spectrum obtained with MODS1. Two components are clearly visible at $\sim 7045 \AA$ and are well separated. The lower component is the quasar J0256+0019 included in our Stripe 82 survey. The only obvious feature in the galaxy spectrum (upper component) is prominent $\mathrm{Ly} \alpha$ emission.

One-dimensional (1D) spectra of both components were obtained using optimal extraction (Horne 1986) while allowing for multiple, blended object profiles (Hynes 2002). We used the direct image obtained immediately following the spectroscopic observations to obtain the profile weights for extraction. We fit a Moffat profile to the quasar and an exponential profile to 

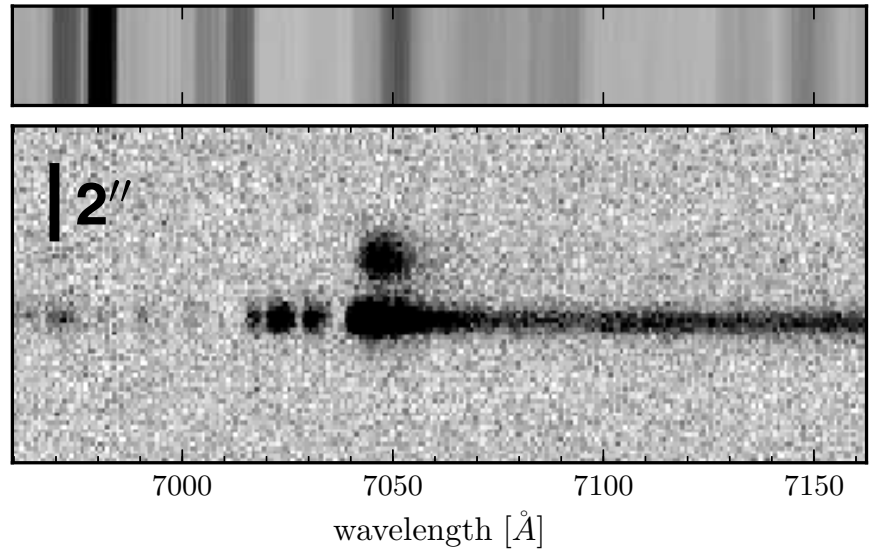

Figure 4. Two-dimensional LBT/MODS spectrum showing the Ly $\alpha$ emission region (seeing $\sim 0^{\prime \prime} 8$ ). The image in the top panel is the model for the sky background, and in the bottom panel the sky-subtracted spectrum. Transmission through the $\operatorname{Ly} \alpha$ forest as well as the rest-frame UV continuum redward of Ly $\alpha$ are visible for the quasar, while the companion presents only strong Ly $\alpha$ emission.

the galaxy, then collapsed the profile fits along the slit axis. The results are similar to those obtained with a simple boxcar extraction, but with lower noise as expected.

Flux calibration is provided by observations of the spectrophotometric standard star GD71. This calibration is affected by the non-photometric conditions and slit losses. We obtain rough corrections for the slit losses using the direct imaging obtained immediately after the spectroscopic observations, by calculating the ratio of the synthetic fluxes obtained from integrating the spectra to the total fluxes measured from the $i$-band imaging. We adopt these corrections ( 0.65 for the quasar and 0.60 for the galaxy), but note that systematic effects may introduce additional (unaccounted for) uncertainty into these values.

Figure 5 displays the extracted spectra of both components, with slit loss corrections applied. The quasar spectrum has typical emission and absorption features for a high-redshift quasar. The galaxy spectrum is dominated by $\mathrm{Ly} \alpha$ emission. Although it is not immediately apparent from Figure 5, we also

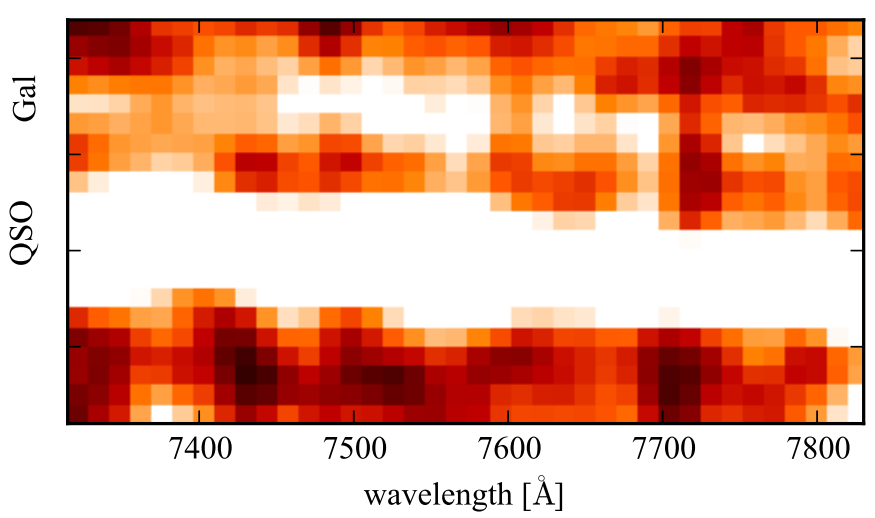

Figure 6. Two-dimensional LBT MODS spectrum highlighting the faint continuum detected from the companion galaxy. The spectrum has been rebinned by a factor of two along the spatial axis $\left(0^{\prime \prime} .25\right.$ pixel $\left.^{-1}\right)$ and a factor of 16 along the spectral axis (14 ̊ pixel $\left.{ }^{-1}\right)$. The image has been further smoothed by a Gaussian filter with $\sigma=1$ pixel to enhance the faint continuum emission from the companion galaxy.

(A color version of this figure is available in the online journal.)

detect continuum flux from the companion galaxy. Figure 6 presents a collapsed version of the 2D spectrum, binning pixels to enhance the $\mathrm{S} / \mathrm{N}$ until the weak continuum of the companion is apparent $\left(\mathrm{S} / \mathrm{N} \sim 0.5\right.$ pixel $\left.^{-1}\right)$. The non-zero continuum level is also evident in the extracted $1 \mathrm{D}$ spectrum.

\subsection{Analysis}

Based on the MMT spectrum alone we considered the possibility that the companion represents a secondary image of a single source quasar due to gravitational lensing, and is visible only in Ly $\alpha$ emission as this was the strongest feature in the quasar spectrum. However, the quasar's C IV line is sufficiently strong that it should have been detected from the companion galaxy if its spectrum were simply scaled from the quasar's spectrum. This conclusion is even stronger based on the higher S/N LBT observations: the flux ratios of the two spectra at the peak of the $\operatorname{Ly} \alpha$ emission are $\sim 2: 1$, compared to a ratio

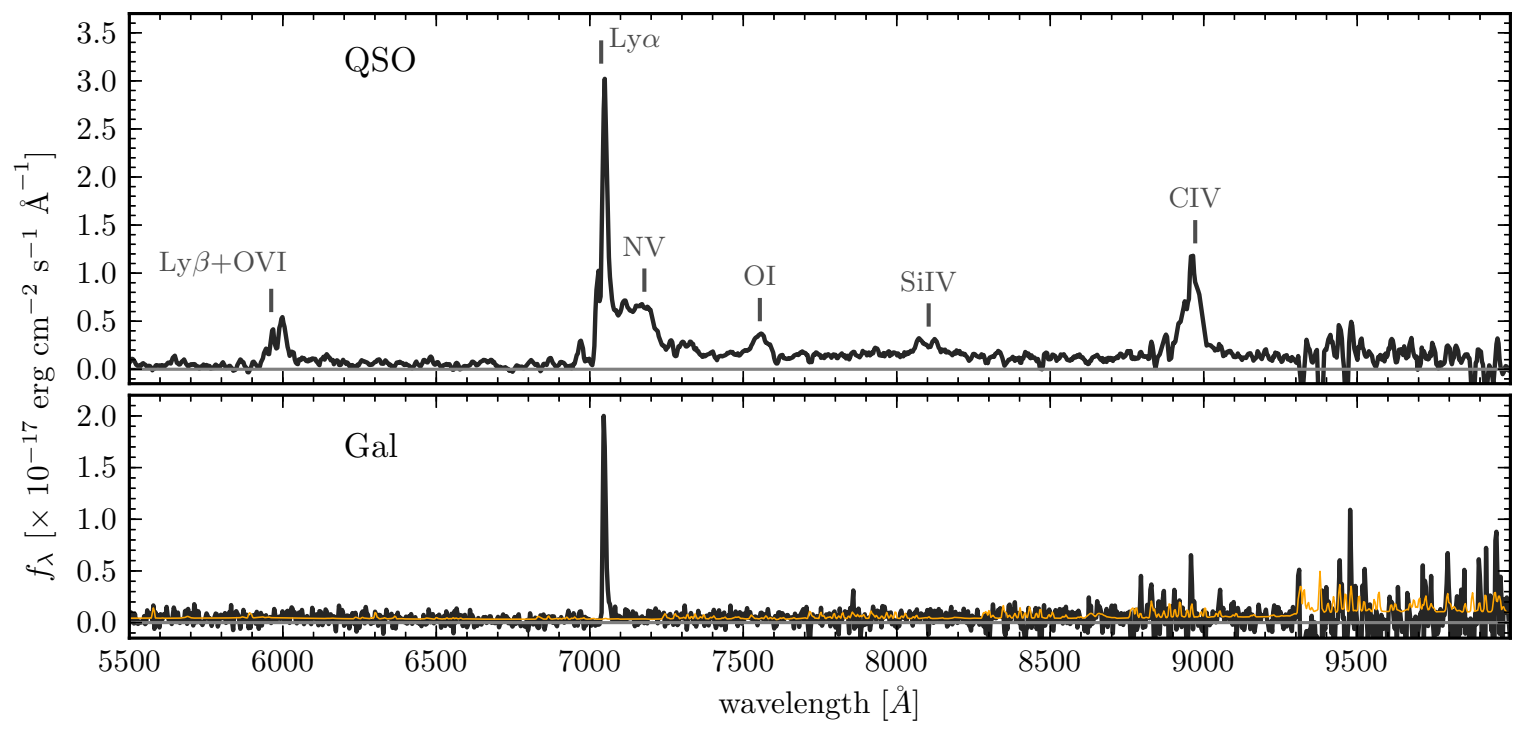

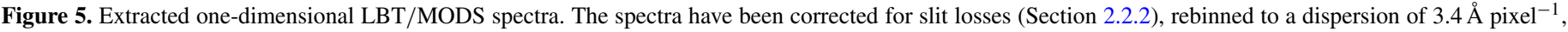

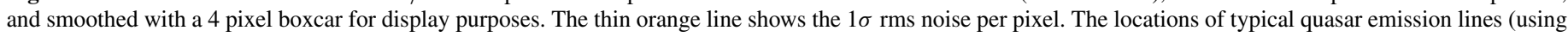
a redshift derived from a Gaussian fit to the $\mathrm{O}_{\mathrm{I}}$ line) are marked in the quasar spectrum.

(A color version of this figure is available in the online journal.) 


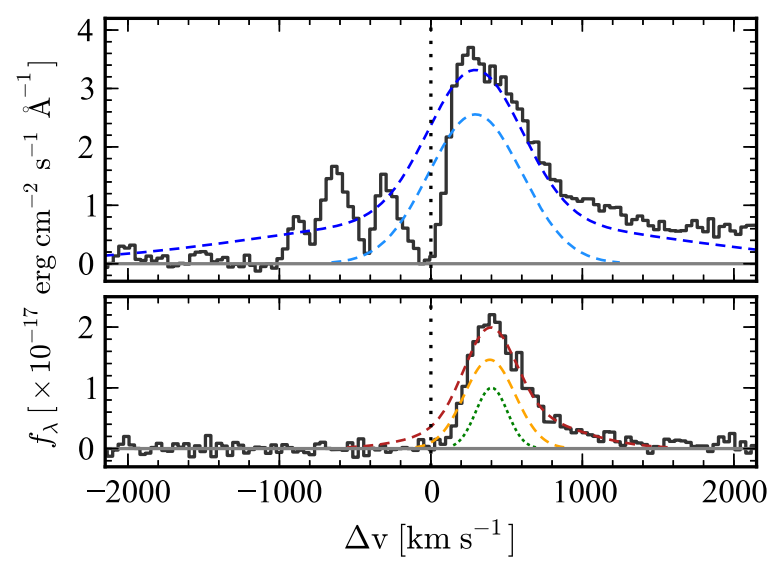

Figure 7. Extracted one-dimensional LBT/MODS spectra showing the Ly $\alpha$ emission regions of the quasar (upper panel) and companion galaxy (lower panel). The slit loss corrections have been applied, but these spectra are not smoothed or binned. Zero velocity is defined using the redshift obtained from the quasar's O I emission line. The dashed lines show the multiple Gaussian fits for both objects (see Table 1), with the lower line showing the narrow component fit and the upper line the sum of the narrow and broad components. The fit to the quasar line profile underestimates the total flux, especially in the presence of the strong absorption features blueward of the line peak. The fit to the galaxy line profile captures the red wing that extends over $\sim 1000 \mathrm{~km} \mathrm{~s}^{-1}$. The observed peaks of the quasar and galaxy Ly $\alpha$ lines are separated by $\sim 150 \mathrm{~km} \mathrm{~s}^{-1}$, although the intrinsic emission peak for the quasar may be significantly bluer and strongly absorbed, implying a larger velocity offset between the two objects. The instrumental resolution is $\sim 230 \mathrm{~km} \mathrm{~s}^{-1}$ (green dotted line in lower panel). (A color version of this figure is available in the online journal.)

at the $C_{\text {IV }}$ peak of $\gtrsim 10: 1$ and a continuum ratio of $\sim 2.5: 1$. Furthermore, the companion galaxy is resolved in the LBT imaging, and no candidate lens galaxy is apparent. Finally, the detailed Ly $\alpha$ profiles show clear differences (see Figure 7). Thus we reject the lensing hypothesis for this object.

Our main results are the detection of strong Ly $\alpha$ emission as well as rest-frame UV continuum from the companion galaxy. By fitting a power law to the spectrum redward of $\operatorname{Ly} \alpha$, we obtain $f_{1500}=(4.7 \pm 0.2) \times 10^{-19} \mathrm{erg} \mathrm{s}^{-1} \mathrm{~cm}^{-2} \AA^{-1}$ (after applying the slit loss correction). This flux density corresponds to a continuum luminosity of $M_{1500}=-22.7$, or $\approx 5.5 L^{*}$ at this redshift (Bouwens et al. 2014). Although this value is high compared to local non-active galaxies, objects with even higher UV luminosities have been found at $z \sim 3$ (Bian et al. 2012) and at $z \sim 7$ (Bowler et al. 2014).

We measure a power-law slope of $\beta_{\lambda}=-1.1 \pm 0.3\left(f_{\lambda} \propto\right.$ $\lambda^{\beta_{\lambda}}$ ) by fitting the UV continuum. This value is quite uncertain given the low $\mathrm{S} / \mathrm{N}$ and the limited wavelength coverage. We fit the power-law continuum of the quasar and obtain a slope $\alpha_{v}=-0.5\left(f_{v} \propto v^{\alpha_{v}}\right)^{9}$. This agrees well with typical UV slopes measured for quasars (e.g., Vanden Berk et al. 2001) and suggests that the relative flux calibration is reliable. A slope $\beta_{\lambda}=-1.1$ is fairly red, but consistent with measurements of galaxies at $z \sim 5$, especially given that the spectral slope becomes redder at higher luminosities (Bouwens et al. 2011).

We measure a redshift of $z=4.789 \pm 0.005$ from a single Gaussian fit to the OI emission line of the quasar. This is a low ionization transition which normally has smaller systematic redshift offsets than lines such as C IV (e.g., Shen et al. 2011); however, we adopt a conservatively large uncertainty on the redshift to account for a possible shift of the O I peak relative

\footnotetext{
9 We follow the convention of measuring quasar power law slopes in frequency units and galaxy slopes in wavelength units.
}

to the systemic velocity. We do not attempt to define a separate redshift for the companion galaxy, as the only detected line is Ly $\alpha$, from which it is notoriously difficult to obtain a reliable redshift; however, the Ly $\alpha$ redshifts for the quasar and galaxy agree to $<200 \mathrm{~km} \mathrm{~s}^{-1}$, suggesting that the true separation of the two objects is not much larger than the projected separation of $12 \mathrm{kpc}$.

Figure 7 shows the Ly $\alpha$ profiles of both the quasar and companion galaxy. The companion galaxy has strong Ly $\alpha$ emission, with $f_{\mathrm{Ly} \alpha}=2.4 \times 10^{-16} \mathrm{erg} \mathrm{s}^{-1} \mathrm{~cm}^{-2} \AA^{-1}$ and $\mathrm{EW}_{0} \sim$ $100 \AA$. The equivalent width is not atypical when compared to high-redshift Ly $\alpha$ emitters (LAEs) selected from narrow-band surveys (e.g., Kashikawa et al. 2011); however, the line strength tends to decrease with continuum luminosity in these objects; thus the galaxy stands out both for its bright continuum and the equivalent width of the $\operatorname{Ly} \alpha$ emission line. The line luminosity is $\sim 6 \times 10^{43} \mathrm{erg} \mathrm{s}^{-1}$; if the Ly $\alpha$ emission were entirely due to star formation, this would correspond to $\operatorname{SFR}(\operatorname{Ly} \alpha) \sim 60 M_{\odot} \mathrm{yr}^{-1}$ using the Kennicutt (1998) relation for $\mathrm{H} \alpha$ and assuming Case B recombination. This is likely to be an underestimate due to intergalactic medium attenuation and dust extinction, both of which would dampen the observed $\operatorname{Ly} \alpha$ line flux.

On the other hand, the $\mathrm{Ly} \alpha$ line profile is unusual when compared to LAEs. From Figure 7 it is evident that the red wing of the line profile extends over a range $|\Delta v| \sim 1000 \mathrm{~km} \mathrm{~s}^{-1}$. A fit to the line with a single Gaussian results in FWHM $700 \mathrm{~km} \mathrm{~s}^{-1}$; a fit with a double Gaussian has a broad wing with FWHM $\sim 1000 \mathrm{~km} \mathrm{~s}^{-1}$. Ohyama et al. (2004) observed a similar feature in the spectrum of one of the Ly $\alpha$ companions to BR 1202-0725. They attributed the broad linewidth to outflows of neutral hydrogen driven by star formation. Given the high estimated star formation rate (SFR) from Ly $\alpha$ (roughly four times that of BR 1202-0725 Ly $\alpha-1$ ) this scenario is plausible.

In Table 1 we provide values obtained from Gaussian fitting of the emission lines in both spectra. For the quasar Ly $\alpha$ line we restrict the fitting to the red wing of the line in order to mask the strong absorption features in the blue wing. Because of the difficulty in fitting the $\operatorname{Ly} \alpha$ profile we do not provide formal uncertainties; similarly, the C IV line is in a region with substantial night sky line residuals and is thus difficult to fit reliably.

The Ly $\alpha$ emission from the quasar is unusually narrow. We measure a FWHM of $\sim 750 \mathrm{~km} \mathrm{~s}^{-1}$ from a single Gaussian fit to the line. This is the narrowest line of the $35 z \sim 5$ quasars from our MMT observations from which we can measure the Ly $\alpha$ line (the median is $\sim 2000 \mathrm{~km} \mathrm{~s}^{-1}$ with a standard deviation of $\sim 500 \mathrm{~km} \mathrm{~s}^{-1}$; only one other object has FWHM $<1000 \mathrm{~km} \mathrm{~s}^{-1}$ ). The Ly $\alpha$ line alone would qualify this object as a Type II quasar candidate according to the criteria of Alexandroff et al. (2013). On the other hand, the Civ line is broader (FWHM $\sim 2000 \mathrm{~km} \mathrm{~s}^{-1}$ ) and the UV continuum is strong $\left(M_{1450} \approx-24\right)$, indicating at best a moderate amount of nuclear extinction. It is evident from Figure 7 that the Ly $\alpha$ line has multiple strong absorption features; this likely explains the narrowness of the line. We re-fit the line using only the red wing and obtain FWHM $\sim 1400 \mathrm{~km} \mathrm{~s}^{-1}$. Although we do not attempt to model the $\operatorname{Ly} \alpha$ absorption in detail, the implication is that $\mathrm{J} 0256+0019$ has a high covering fraction of neutral gas along the line of sight when compared to quasars at a similar redshift. A virial estimate for the $\mathrm{BH}$ mass from the $\mathrm{C}$ IV line width using the relation of Vestergaard \& Peterson (2006) gives a mass of $\approx 1.2 \times 10^{8} M_{\odot}$ and an Eddington ratio of $\lambda \approx 0.9$, indicating that the quasar is in a phase of rapid growth. 
Table 1

Properties of J0256+0019

\begin{tabular}{|c|c|c|}
\hline & QSO & Gal \\
\hline R.A. (J2000) & $02: 56: 17.741$ & 02:56:17.804 \\
\hline Decl. (J2000) & $+00: 19: 03.92$ & $+00: 19: 05.49$ \\
\hline$g$ (Stripe 82) & $>26.0$ & $>25.0$ \\
\hline$r$ & $23.75 \pm 0.09$ & $>24.5$ \\
\hline$i$ & $21.88 \pm 0.02$ & $23.6 \pm 0.2$ \\
\hline$z$ & $21.93 \pm 0.10$ & $>22.5$ \\
\hline$i(\mathrm{LBT})$ & $22.11 \pm 0.02$ & $23.62 \pm 0.09$ \\
\hline$J(\mathrm{MMT})$ & $22.11 \pm 0.14$ & $>22.8$ \\
\hline$H$ & $21.43 \pm 0.10$ & $>23.2$ \\
\hline$m_{8700}$ & 24.7 & 25.6 \\
\hline$M_{1500}$ & -23.6 & -22.7 \\
\hline $\operatorname{SFR}(\mathrm{UV})\left(M_{\odot} \mathrm{yr}^{-1}\right)$ & $\cdots$ & 13 \\
\hline$z_{\text {Ly } \alpha}{ }^{\mathrm{a}}$ & 4.794 & 4.796 \\
\hline$f_{\mathrm{Ly} \alpha}\left(\operatorname{erg~s}^{-1} \mathrm{~cm}^{-2} \AA^{-1}\right)$ & $\cdots$ & $24 \times 10^{-17}$ \\
\hline$L_{\mathrm{Ly} \alpha}\left(\operatorname{erg~s}^{-1}\right)$ & $\cdots$ & $58 \times 10^{42}$ \\
\hline $\operatorname{SFR}(\operatorname{Ly} \alpha)\left(M_{\odot} \mathrm{yr}^{-1}\right)$ & $\cdots$ & 60 \\
\hline $\mathrm{EW}_{0}(\mathrm{Ly} \alpha)(\AA)$ & 120 & 100 \\
\hline ...broad & 53 & 38 \\
\hline ...narrow & 65 & 42 \\
\hline $\operatorname{FWHM}(\operatorname{Ly} \alpha)\left(\mathrm{km} \mathrm{s}^{-1}\right)$ & 1380 & 690 \\
\hline ...broad & 3010 & 970 \\
\hline ...narrow & 720 & 400 \\
\hline $\mathrm{EW}_{0}(\mathrm{~N} v)(\AA)$ & $\cdots$ & $<0.2$ \\
\hline $\mathrm{EW}_{0}\left(\mathrm{C}_{\mathrm{IV}}\right)(\AA)$ & 75 & $<5.7$ \\
\hline
\end{tabular}

Notes. All photometry is on the AB system and corrected for Galactic extinction. Stripe 82 photometry for the quasar is derived from the coadded imaging. The $g$-band upper limit is the $3 \sigma$ limit for point sources in the field. We fit the companion galaxy with a 2D elliptical Gaussian using GALFIT and a PSF model derived from stars in the field using DAOPHOT. This model was applied to the other bands to derive $3 \sigma$ upper limits. All flux-based quantities derived from the spectra have been corrected for slit losses (see Section 2.2.2).

${ }^{a}$ Redshift measured from the narrow component of the double Gaussian fit to the Ly $\alpha$ line.

No rest-frame UV emission lines other than Ly $\alpha$ are detected from the companion galaxy; thus it is unlikely to be an active galactic nucleus (AGN). Figure 8 shows the $\mathrm{C}$ IV emission region of both the quasar and the companion. We obtain an upper limit on the C IV emission from the companion galaxy by using the redshift obtained from the quasar $\mathrm{O}_{\mathrm{I}}$ line to set the wavelength and a fiducial linewidth of $\sigma_{\mathrm{v}}=200 \mathrm{~km} \mathrm{~s}^{-1}$. This limit is $\mathrm{EW}_{0}<6 \AA(1 \sigma)$, whereas nearly all of the Type II quasar candidates in Alexandroff et al. (2013) have $\mathrm{EW}_{0}(\mathrm{C}$ IV) $>$ $10 \AA$. Additionally, no N v emission is detected from the galaxy, to a limit of $\mathrm{EW}_{0}(\mathrm{Nv})<0.2 \AA$. These properties are similar to the Ly $\alpha-1$ companion galaxy of BR 1202-0725, which Williams et al. (2014) found to have ratios of C IV flux to Ly $\alpha$ flux $<0.033$ and $\mathrm{N}$ IV $/$ Ly $\alpha<0.019$, compared to $>0.2$ and $\gtrsim 0.1$, respectively, for typical Seyfert galaxies and obscured quasars. These ratios are $\approx 0.05$ and $<0.001$ for the J0256+0019 companion, suggesting that AGN photoionization plays at best a minor role.

\section{J0050+3445}

The $z \sim 6$ quasar J0050+3445 was discovered as part of the Canada-France High- $z$ Quasar Survey (CFHQS; Willott et al. 2010b). Compared to the bright $z \sim 6$ quasars discovered in the SDSS main survey (e.g., Fan et al. 2001), the CFHQS quasars are selected from smaller area, deeper imaging from various CFHT survey fields, and thus tend to be fainter. However,

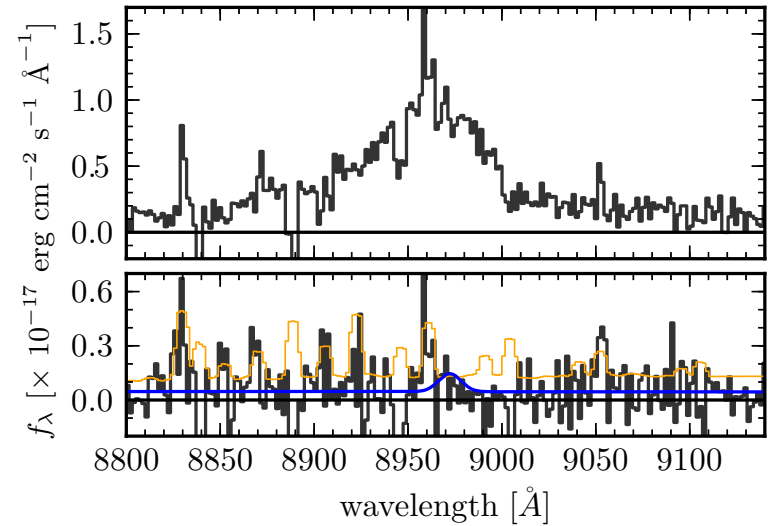

Figure 8. Extracted one-dimensional LBT/MODS spectra showing the C IV emission region (rebinned to $1.6 \AA \mathrm{pixel}^{-1}$ and corrected for slit losses). The upper panel displays the quasar spectrum and the lower panel the companion galaxy, along with the rms noise (thin orange line). The bottom panel also presents the fit to the galaxy continuum as a solid blue line, including a Gaussian profile to represent the $1 \sigma$ upper limit on the $\mathrm{C}$ IV emission $\left(\mathrm{EW}_{0}<6 \AA\right.$ ). This limit assumes the redshift obtained from the quasar's O I line and a width of $200 \mathrm{~km} \mathrm{~s}^{-1}$ (the quasar's C IV line is blueshifted, which is a common feature in quasar spectra; cf. Richards et al. 2011). C IV falls within a noisy portion of the $\mathrm{OH}$ forest and there are significant residuals from the sky line subtraction; however, it is evident the galaxy does not have strong CIV emission. The apparent feature at $\sim 8960 \AA$ lies on a strong sky emission line; a similar feature is visible in the quasar spectrum and thus it is not likely to be real.

(A color version of this figure is available in the online journal.)

$\mathrm{J} 0050+3445$ is one of the brightest quasars in the CFHQS, with $z_{\mathrm{AB}}=20.5$. It is also one of the most distant objects, with $z=6.253 \pm 0.003$ measured from the Mg II line (Willott et al. 2010a). The Mg II line was also used for a virial estimate of the $\mathrm{BH}$ mass, $M_{\mathrm{bh}}=2.6 \times 10^{9} M_{\odot}$. The mass and Eddington ratio $\left(\lambda_{\text {Edd }}=0.6\right)$ are typical of bright $z \sim 6$ quasars.

\subsection{HST Observations}

We have been performing a systematic search for evidence of gravitational lensing among $z \sim 6$ quasars through an HST SNAP program (\#12184, PI: X. Fan). This program includes nearly all of the known $z \sim 6$ quasars and utilizes WFC3/IR F105W imaging. Full results of the SNAP study will be presented in a forthcoming work (I. D. McGreer et al., in preparation). During the SNAP program J0050+3445 was observed with two $180 \mathrm{~s}$ exposures. It was immediately apparent that the resulting image was inconsistent with a single pointspread function (PSF) detection, as there was excess flux detected at $\sim 1^{\prime \prime}$ from the quasar position. However, the shallow, single-band SNAP observations did not constrain the nature of the excess emission, which could be attributed to (1) a foreground interloper, (2) a high-redshift galaxy, or (3) a secondary lensed image of the quasar.

\subsubsection{HST Cycle 19 Imaging}

To further explore the nature of the excess flux in the SNAP imaging we obtained additional HST imaging of J0050+3445 in Cycle 19 (\#12493, PI: I. D. McGreer). These observations consisted of two components: (1) three orbits of ACS/WFC imaging with the F775 bandpass, and (2) two orbits of WFC3/ IR imaging with the F105W bandpass. This filter combination isolates objects at the redshift of the quasar by taking advantage of the strong spectral break feature introduced by the nearly saturated Ly $\alpha$ forest absorption at $z=6.25$. A single broadband color can provide strong evidence that a detected object is at high 


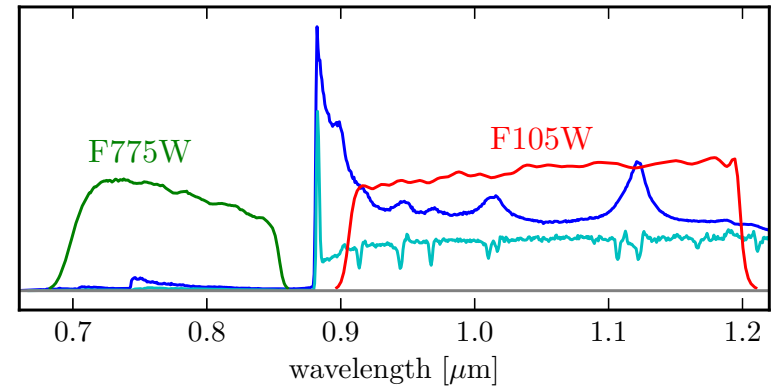

Figure 9. HST filter set for the J0050+3445 observations. The labeled green and red lines show the F775W and F105W transmission curves, respectively. A template quasar spectrum (Vanden Berk et al. 2001) at $z=6.25$ is shown in blue, and a template Lyman break galaxy (Shapley et al. 2003) is shown in cyan. Both templates have had Ly $\alpha$ forest absorption applied using a mean transmission spectrum from our simulations (Section 3.4). Ly $\alpha$ emission at this redshift falls between the two filters.

(A color version of this figure is available in the online journal.)

redshift if the filters straddle the Lyman break. Figure 9 displays the filter choices for the HST observations with spectral energy distributions (SEDs) of a high-redshift quasar and galaxy for reference. Neither of our filters includes Ly $\alpha$ emission at the redshift of the quasar.

The ACS/WFC imaging occurred on 2012 June 13. The three orbits were divided into two exposures, the first with four dither positions and the second with two dither positions. Images were processed using AstroDrizzLE. First, the standard pipelinereduced images were combined into two drizzled images, which were then aligned with the TWEAKSHIFTs routine. After propagating the calculated offsets to the input images, they were combined into a single drizzled image. The full integration time in the final mosaic is $8072 \mathrm{~s}$. We used pixfrac $=0.8$ and a scale of 0 '.03 for the output pixel grid (Koekemoer et al. 2011).

The WFC3/IR imaging occurred on 2012 June 8. A similar dither pattern was employed. Although cosmic rays in individual images can be rejected by the continuous sampling of the detector array, we found some residual hot pixels in the individual images. We thus used TWEAKREG and AsTroDrizZLE in a single iteration with cosmic ray rejection enabled to produce a final drizzled image combining all six individual images, with a total integration time of $5018 \mathrm{~s}$. We used pixfrac $=0.8$ and a scale of 0 '.06 for the final pixel grid.

We found small $\left(<1^{\prime \prime}\right)$ offsets between the final ACS and WFC3 mosaics when compared to SDSS imaging over the same area. We thus aligned the ACS image to the SDSS DR9 (Ahn et al. 2012) $i$-band image of the field using TWEAKSHIFTs, and then aligned the WFC3 image to the corrected ACS image. The final astrometric accuracy is $<0$ '! 1 when compared to SDSS.

\subsection{Image Decomposition}

Figure 10 presents the HST imaging obtained for J0050+3445. Within the central $\sim 1^{\prime \prime}$ the ACS image contains only a single faint detection corresponding to the $z=6.25$ quasar, while the WFC3 image includes both the bright quasar PSF component and an additional extended component (a galaxy), consistent with the results from the shallow SNAP imaging.

We first construct PSF models in order to decompose the images. For both the ACS and WFC3 images we generate empirical PSF models from stars within the field using IRAF DAOPHOT tasks. Analysis of the ACS image is somewhat insensitive to the accuracy of the PSF model, as the quasar is relatively weak and the galaxy lies well beyond the extent of the quasar's light profile. On the other hand, the quasar is much brighter in the WFC3 image, and positive flux from the wings of the PSF extends to the position of the galaxy. We selected eight isolated stars with high $S / N$ detections to construct the WFC3 PSF model. We also experimented with TinyTim PSF models, but found they were not an improvement over the empirical PSF (cf. Mechtley et al. 2012).

We next use GALFIT to model the quasar and galaxy in the HST images. The ACS image is fit with a single PSF component to represent the quasar. The model for the WFC3 image includes both a PSF component for the quasar and an exponential disk model for the galaxy. The exponential disk profile was selected empirically; we fix the axis ratio and position angle to values obtained from visually matching the galaxy profile in order to reduce the fit degeneracies. Finally, we find that the PSF subtraction is improved by masking the core of the quasar PSF during the fit. We mask the central $3 \times 3$ pixels $\left(0{ }^{\prime} .18\right)$. The results of the GALFIT fitting are given in Table 2.

\section{ACS/F775W}

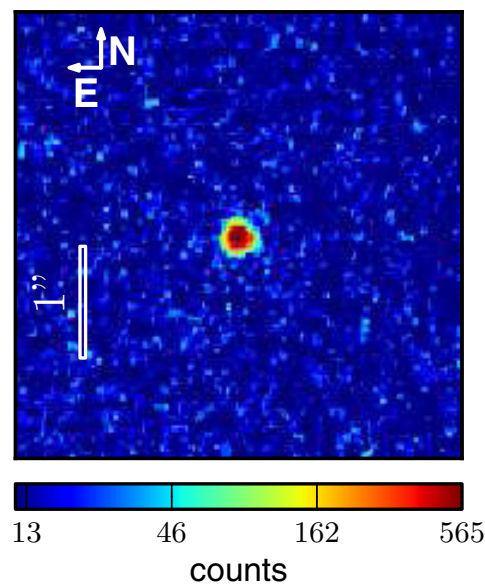

WFC3/F105W

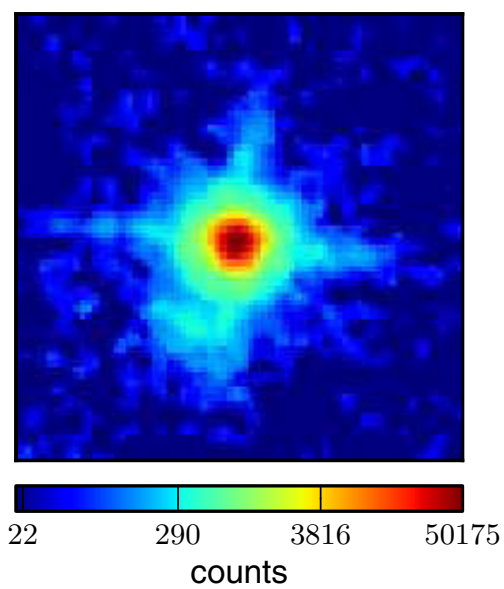

WFC3 PSF sub.

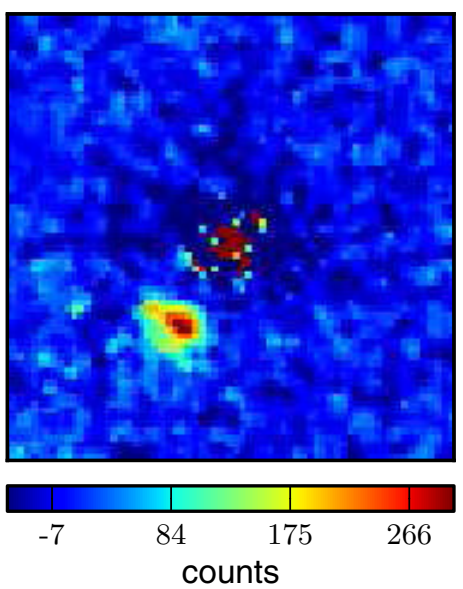

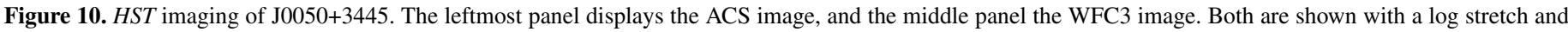

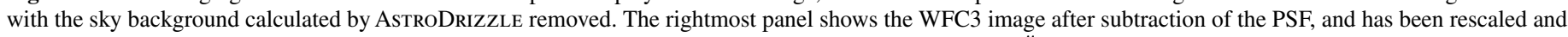
placed on a linear stretch to highlight the galaxy. The companion has $Y_{105}=25.0$ and is at a separation of $0^{\prime \prime} 6$ from the quasar.

(A color version of this figure is available in the online journal.) 
Table 2

Properties of J0050+3445

\begin{tabular}{lcc}
\hline \hline & QSO & Gal \\
\hline$\Delta \alpha$ & 0.00 & $+0^{\prime \prime} .45$ \\
$\Delta \delta$ & 0.00 & $-0^{\prime \prime} 74$ \\
$i_{775}$ & $24.032 \pm 0.008$ & $>26.9$ \\
$Y_{105}$ & $20.325 \pm 0.003$ & $25.06 \pm 0.23$ \\
$r_{s}$ & $\ldots$ & $0.09 \pm 0.03$ \\
$a / b$ & $\ldots$ & 0.4 \\
P.A. & $\ldots$ & 63.5 \\
$M_{1500}$ & $-26.64^{\mathrm{a}}$ & -21.76 \\
SFR(UV) & $\ldots$ & $27 M_{\odot} \mathrm{yr}^{-1}$ \\
\hline
\end{tabular}

Notes. Measured quantities are from GALFIT. The axis ratio $(a / b)$ and position angle (P.A.) were fixed during fitting. P.A. is measured east of north.

${ }^{a}$ From Willott et al. (2010b).

The neighboring galaxy is not formally detected in the ACS image. We obtain an upper limit for its flux by first subtracting the PSF component and then fitting the residual image with a model for the galaxy. The parameters for the galaxy model are fixed to the values obtained from fitting the WFC3 image. We then increase the flux in the model galaxy until the fit results in $\Delta\left(\chi^{2}\right)=1$ compared to the PSF-only fit. We adopt this value as the upper limit for the $i_{775}$ flux of the galaxy given in Table 2. The limit obtained from this method, $i_{775}>26.9$, is bright compared to the average depth of the ACS image. This results from a slight positive fluctuation at the position of the galaxy in the ACS image $\left(\sim+1 \sigma\right.$ pixel $^{-1}$ for several neighboring pixels). However, this fluctuation appears to be associated with cosmic ray hits that appear in two out of the six ACS frames. We insert fake galaxies at random locations around the quasar position at the same radius of the neighboring galaxy and then repeat the process for deriving an upper limit. We find that in general the limiting flux is $i_{775}>28$. Assuming that the positive flux is simply a noise fluctuation, the lower limit on the color is $i_{775}-Y_{105} \gtrsim 3(1 \sigma)$, consistent with a $z=6.25$ galaxy. However, we adopt the more conservative limit obtained at the galaxy's position in the image, yielding a color $i_{775}-Y_{105}>1.8$.

\subsection{Analysis}

The original goal of the SNAP program was to search for gravitational lenses, and one of the primary goals of the subsequent imaging was to test the lensing hypothesis for $\mathrm{J} 0050+3445$. As one of most luminous quasars in the CFHQS, with $M_{1450}=-26.6$, and given the excess emission detected in the SNAP imaging, J0050+3445 was a prime candidate for a gravitationally lensed quasar. However, the Cycle 19 HST imaging rules out the lensing hypothesis, at least at $\gtrsim 0^{\prime \prime}$. 1 scales, and thus suggests the measured luminosity is intrinsic. First, the secondary component is extended. While this could be a lens galaxy, no additional lensed quasar images are detected. The limit obtained from the ACS imaging is even more stringent, with higher resolution and easier image decomposition. The quasar is detected at $\sim 200 \sigma$, and the detection limit for point sources is $i_{775} \sim 29$, so flux ratios $\lesssim 60: 1$ can be excluded at $\gtrsim 0$.'05 separations. These constraints effectively rule out all of the parameter space expected for strong lensing of high-redshift quasars (Turner et al. 1984; Comerford et al. 2002; Richards et al. 2004); even the inclusion of ellipticity or shear to the lens model will generally result in strong lensing with multiple images that should be apparent with HST's resolution and depth (Keeton et al. 2005).

The neighboring galaxy is also unlikely to be a foreground interloper or even a high-redshift galaxy unassociated with the quasar. The non-detection in the ACS imaging indicates an extremely red $i-Y$ color, akin to Lyman break galaxies (LBGs) at $z>6$. To examine the significance of the $i-Y$ color, we compare to the CANDELS GOODS-South compilation of Guo et al. (2013), which includes ACS-i $i_{775}$ imaging from GOODS (Giavalisco et al. 2004) and WFC3- $Y_{105}$ imaging from CANDELS (Grogin et al. 2011). We examine the distribution of $i_{775}-Y_{105}$ colors for objects within the GOODS-S area with coverage in both filters, amounting to an area of $\sim 70 \mathrm{arcmin}^{2}$. We make conservative cuts of $Y_{105}<25$ and $i_{775}-Y_{105}>2.0$, finding a density of $\sim 1 \mathrm{arcmin}^{-2}$. At this density, the probability of finding a galaxy similar to the J0050+3445 companion within $1^{\prime \prime}$ of the quasar by chance is $\sim 8 \times 10^{-4}$. This is strong evidence that the neighboring galaxy is indeed associated with the quasar. The projected separation between the two objects is $5.0 \mathrm{kpc}$ if they are at the same redshift.

The Ly $\alpha$ line falls outside of both the F775W and F105W bandpasses (Figure 9). The detected emission is most likely dominated by UV continuum, while the presence of strong Ly $\alpha$ emission - as in the case of J0256+0019-is unconstrained by our observations. The galaxy is resolved in the WFC3 image, with a fitted disk scale length of $0^{\prime \prime} .09$, or $0.5 \mathrm{kpc}$ at $z=6.25$. The derived UV luminosity is $M_{1500}=-21.8$, which is $\approx 5 L^{*}$ at this redshift (e.g., McLure et al. 2013; Schenker et al. 2013), and is comparable to the brightest galaxies found in ground-based surveys at this redshift (Bowler et al. 2012).

A possible explanation for the high UV luminosity of the J0050+3445 companion galaxy is that it is powered by an AGN. At lower redshift, the fraction of galaxies hosting an AGN increases steeply with the host luminosity (e.g., Juneau et al. 2013). In fact, the derived UV luminosity of the J0050+3445 companion is comparable to that of Seyfert galaxies. The possibility of an AGN is intriguing, as it would imply two massive BHs at $z=6.25$ separated by $<10 \mathrm{kpc}$ and potentially merging in a brief timescale. However, the currently available data do not provide any indicators to assess the presence of a low-luminosity AGN in the companion galaxy, other than to note that its morphology does not appear to be dominated by a central point source. Obtaining ground-based spectroscopy of the companion would be challenging, given its small separation from the nearby luminous quasar and its faintness.

\subsection{Search for Additional Neighboring Galaxies}

Our two-band imaging was designed to provide information on the excess flux detected $<1^{\prime \prime}$ from the quasar, but also allows color selection of neighboring galaxies over the entire field. We thus search for associated galaxies up to $\sim 2^{\prime}$ from the quasar. The total area of overlap between the ACS and WFC3 imaging is $4.9 \mathrm{arcmin}^{2}$. We construct matched catalogs by first aligning the ACS image to the WFC3 image with AstroDrizZLE and then executing SExtractor in dual-image mode with the WFC3 image for object detection.

We use simulations of the Ly $\alpha$ forest (see McGreer et al. 2013) and a simple power-law model for the UV continuum from galaxies with a range $-2.5<\beta_{\lambda}<-1$ to estimate the range of galaxy colors expected at $z=6.25^{10}$. From

\footnotetext{
10 This is a much narrower selection than used in past surveys around $z \sim 6$ quasars, which typically adopted a single, broad color cut that encompasses a large volume at $z>6$.
} 


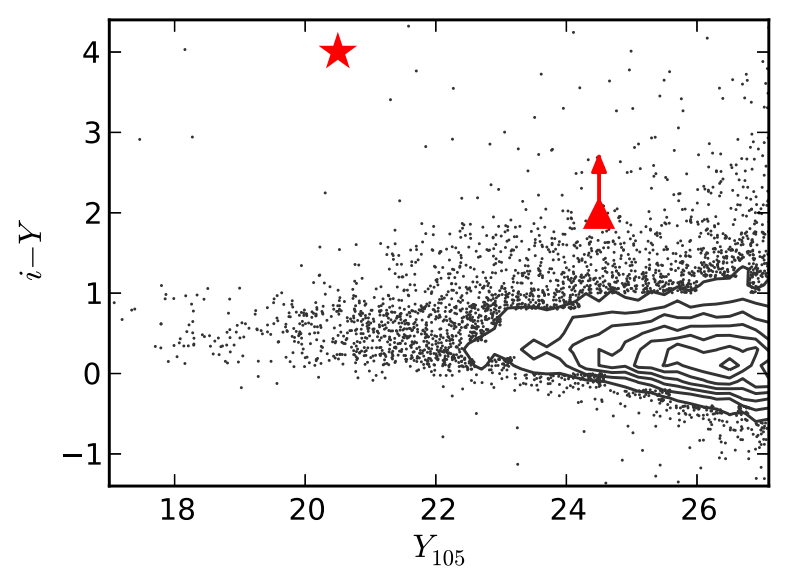

Figure 11. Distribution of $i-Y$ colors for objects from the GOODS-S catalogs of Guo et al. (2013), shown as black contours and points. The area covered by the combined $\mathrm{F} 775 \mathrm{~W} / \mathrm{F} 105 \mathrm{~W}$ imaging is $\approx 14 \times$ greater than for our single WFC3 pointing. The position of the quasar in this plot is represented by a red star, while the position of the companion galaxy (with the conservative lower limit on the $i-Y$ color) is given by the triangle. The very red color of the quasar may be affected by the contribution of the C IV line to the F105W bandpass.

(A color version of this figure is available in the online journal.)

this approach the typical color is $i_{775}-Y_{105} \approx 3.1$, with $i_{775}-Y_{105}>2.8$ for all simulated galaxies. The quasar has $i_{775}-Y_{105}=3.7$. We estimate a $3 \sigma$ detection limit in the ACS image of $i_{775}=28.2$ in a $0^{\prime \prime} .24$ aperture, though the same limit is somewhat weaker using the elliptical apertures defined for the WFC3 detections (i.e., SExtractor MAG_AUTO), for which we obtain $i_{775} \approx 27.8$. We thus survey for associated galaxies by searching for $i_{775}$-dropouts with $Y_{105}<25.0$; more specifically, we require $i_{775}-Y_{105}>2.8$ to the detection limit of $i_{775} \approx 28$.

We identify four candidates from the SExtractor catalogs with these criteria (excluding the J0050+3445 quasar and companion galaxy). Of those objects, three are near the selection limit $\left(Y_{105} \approx 25\right)$ and visual inspection of the ACS images shows they are weakly detected in the optical band. We do not consider them to be good candidates for $z \sim 6.25$ galaxies. The final object has $Y_{105}=24.24 \pm 0.05$ and is also weakly detected in the ACS image. Using a circular $1^{\prime \prime}$ diameter aperture instead of the MAG_AUTO photometry, which roughly matches the size of the object in the WFC3 image, we obtain $i_{775}-Y_{105} \approx 1.9$. We also do not consider this object to be a good candidate for an associated galaxy. From visual inspection we identify one object $11^{\prime \prime} .5$ from the quasar that appears to be a true optical dropout; however, with $Y_{105}=25.4$ it is too faint for the color to be conclusive $\left(i_{775}-Y_{105} \gtrsim 2.6\right)$.

We also broaden our search to all optical dropouts to a depth of $Y_{105}=27$. The ACS non-detections do not provide strong constraints on the color for these objects. However, we can compare the number counts of the faint WFC3 detections to those from the GOODS-S catalogs from CANDELS described in Guo et al. (2013). We restrict the GOODS-S area to regions covered by both ACS/F775W and WFC3/F105W with integration times in each band greater than the integration times in our observations. We then consider any GOODS-S object with $i_{775}>28$ a "dropout," as it would be below the detection limit of our ACS imaging. We find no excess of such objects in our imaging after comparing to the number density obtained from the GOODS-S data (see Figure 11).

The fact that we see no excess of associated galaxies on large scales around the quasar $\mathrm{J} 0050+3445$ is consistent with previous

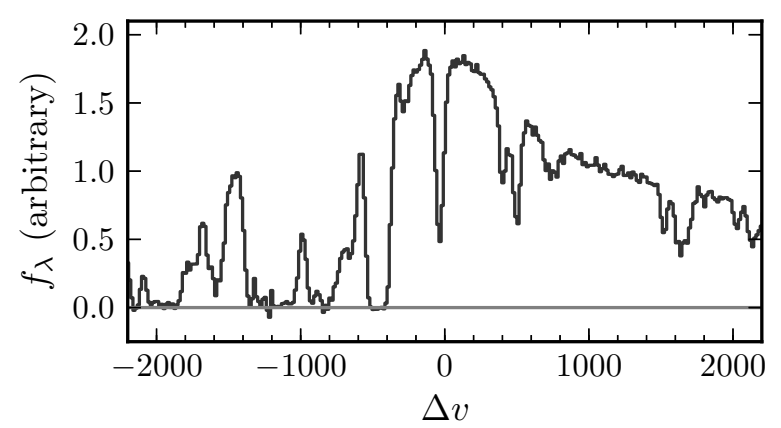

Figure 12. Portion of a $1.7 \mathrm{hr}$ Keck ESI spectrum (Becker et al. 2011) with resolution $\sim 50 \mathrm{~km} \mathrm{~s}^{-1}$ covering the Ly $\alpha$ emission region of J0050+3445. Zero velocity is defined using the Mg II redshift reported by Willott et al. (2010a). There is an absorption system close to zero velocity and a stronger system at $\sim-500 \mathrm{~km} \mathrm{~s}^{-1}$.

HST results that did not find overdensities to be ubiquitous among high-redshift quasars (e.g., Kim et al. 2009). However, using F105W for detection restricts us to objects with bright rest-frame UV continuum; we are not sensitive to obscured sources or weak continuum sources with strong Ly $\alpha$ emission as the line falls outside our bandpass. On the other hand, a depth of $Y_{105}=27$ corresponds to $\approx L^{*}$ at this redshift (McLure et al. 2013; Schenker et al. 2013); thus a strong overdensity of massive, star-forming galaxies should have been detectable with our observations.

\section{DISCUSSION}

\subsection{What Powers the Observed Emission?}

The spectra of both quasars include strong Ly $\alpha$ absorption features near the quasar redshift (Figures 7 and 12). Such features are not uncommon in high-redshift quasar spectra, and may be indicative of a large reservoir of cold neutral gas surrounding the quasar. Further evidence for a high covering fraction of neutral gas in $\mathrm{J} 0256+0019$ comes from its relatively weak and narrow Ly $\alpha$ line. Such halos may give rise to fluorescent Ly $\alpha$ emission (Haiman \& Rees 2001) powered by the quasar's ionizing continuum. Could the companions be dense clouds reprocessing the ionizing flux from the quasars, rather than self-luminous systems?

We consider the contribution of fluorescent $\mathrm{Ly} \alpha$ emission to the observed spectrum of the J0256+0019 companion galaxy by determining whether the incident ionizing flux from the quasar onto the galaxy is sufficient to power its strong Ly $\alpha$ flux. We adopt the projected separation between the two objects and a radius of $3 \mathrm{kpc}$ for the companion (roughly its measured extent from the LBT image). We further assume that the quasar UV emission is isotropic and has a typical UV power-law continuum (Shull et al. 2012). The incident ionizing photon flux on the galaxy is then $\sim 4 \times 10^{54} \mathrm{erg} \mathrm{s}^{-1}$. If every photon resulted in a Ly $\alpha$ photon, the resulting Ly $\alpha$ luminosity would be $\sim 6 \times 10^{43} \mathrm{erg} \mathrm{s}^{-1}$, which is roughly equal to the measured luminosity. In other words, the observed strong $\operatorname{Ly} \alpha$ emission could be due entirely to fluorescence if all of the quasar's ionizing photons incident on the galaxy are re-emitted as Ly $\alpha$ photons by the galaxy, whereas only $\sim 60 \%$ of ionizing photons should result in $\mathrm{Ly} \alpha$ photons even for a single optically thick cloud (Gould \& Weinberg 1996).

The J0256+0019 companion galaxy also has strong continuum emission, with $v L_{v} \approx 10^{45} \mathrm{erg} \mathrm{s}^{-1}$ at rest-frame $1500 \AA$, so its spectrum cannot be purely fluorescent. Similarly, for 
J0050+3445 the observed emission arises only from continuum redward of Ly $\alpha$. The lack of other emission lines in the spectrum of the J0256+0019 companion further demonstrates that neutral gas within its ISM is not being ionized by the nearby quasar (e.g., Gunn 1971; Filippenko 1985; see also the case of Hanny's Voorwerp, a cloud of gas illuminated by a (now dormant) quasar that has strong high ionization lines (Keel et al. 2012)). However, it is intriguing to consider that, at least in the case of $\mathrm{J} 0256+0019$, some of the strong Ly $\alpha$ emission may arise from fluorescence in a neutral halo of metal-poor gas surrounding the galaxy. This interpretation is consistent with the larger SFR inferred from the Ly $\alpha$ emission than the UV continuum (about a factor of four, see Table 1).

The companion galaxies may themselves be AGNs. Indeed, in merger-driven models for high-redshift quasar triggering (e.g., Li et al. 2007) it is expected that the progenitor galaxies each carry their own massive $\mathrm{BH}$, although it may not be likely for both to be active at the same time. We argued in Section 2.3 that the lack of AGN lines in J0256+0019 argues against an AGN in this object. The constraints on AGN activity in the J0050+3445 companion are weaker, although we noted in Section 3.3 that its observed emission is not nuclear-dominated.

While we cannot conclusively rule out AGN activity in either system, the current observations are consistent with both galaxies being powered by internal star formation. We next discuss the implications of finding two unusually bright galaxies in the vicinity of high-redshift quasars.

\subsection{Are These Major Mergers?}

The presence of bright galaxies within $\sim 10 \mathrm{kpc}$ of two luminous, high-redshift quasars is highly suggestive of ongoing major mergers in both systems. Far-IR measurements of C II line widths of $z \sim 6$ quasars have shown that their dynamical masses are roughly $\sim 10^{10-11} M_{\odot}$ (Wang et al. 2013). C II dynamical masses may underestimate the full mass of the stellar bulge if the line emission is mainly concentrated in the center of the host galaxy; however, $\mathrm{CO}$ observations further indicate masses $>10^{10} M_{\odot}$ in molecular gas alone (Wang et al. 2010). This result is consistent with an expectation that the hosts of high-redshift quasars are massive, with the archetypal case being J1148+5251 at $z=6.4$ (Walter et al. 2003).

Next, we consider the galaxy companions. According to the $z=5.7$ Ly $\alpha$ luminosity function from Kashikawa et al. (2011), the J0256+0019 companion galaxy is at $\sim 6 L^{*}$, and thus on the steeply falling bright end of the luminosity function. It can be compared to Himiko, an extremely bright LAE discovered in the Subaru Deep Field (Ouchi et al. 2009). The J0256+0019 companion galaxy has many features in common with Himiko: it is resolved in ground-based imaging, and has a comparable Ly $\alpha$ luminosity (if not somewhat greater), equivalent width, and UV continuum luminosity. Using multiwavelength SED fitting, Ouchi et al. (2009) inferred a stellar mass of $\sim 4 \times 10^{10} M_{\odot}$ for Himiko.

We can compare J0050+3445 to field LBGs selected at a similar redshift. Curtis-Lake et al. (2013) examined luminous $\left(L>1.2 L^{*}\right)$ galaxies with spectroscopic redshifts $5.5<$ $z<6.5$ and found that the stellar masses lie in the range $10^{9}<M_{*}<10^{10} M_{\odot}$, with typical stellar population ages of $50-200$ Myr. At $\approx 5 L^{*}$, the companion to J0050+3445 is comparable to the most extreme objects in the Curtis-Lake et al. (2013) sample, and thus likely lies at the higher mass end of this distribution. Similarly, the stellar mass-UV luminosity relation of Stark et al. (2009) derived at $z \sim 4$ would predict a mass of
$M_{*} \sim 10^{10} M_{\odot}$ at this luminosity; this relation shows little or no evolution to higher redshift (e.g., Curtis-Lake et al. 2013).

Thus, comparison to results from high-redshift surveys implies that both the quasar host galaxies and the companion galaxies have masses $>10^{10} M_{\odot}$. The small projected separations between the quasars and companion galaxies $(\lesssim 10 \mathrm{kpc})$ in both cases place the quasar host and companion within the virial radius of a $10^{11}-10^{12} M_{\odot}$ halo (the mass range of a halo likely to host a luminous quasar at this redshift), such that their eventual coalescence seems inevitable. A likely conclusion, then, is that in both cases we are witnessing extreme major merger events, potentially fueling luminous quasar and powerful starburst activity simultaneously.

\subsection{How Unusual Are These Systems?}

Both of the systems discussed here were discovered serendipitously and it is not straightforward to draw conclusions about how common or rare they may be. However, we can ask whether similar systems would have been detected in the course of our surveys.

J0256+0019 was discovered in slit spectroscopic observations. We observed 55 quasars at $z \sim 5$ with the $180^{\prime \prime}$ longslit on MMT Red Channel during the course of our Stripe 82 survey (McGreer et al. 2013). Roughly half were observed with a $1^{\prime \prime}$ slit, the other half with a $1^{\prime \prime} .5$ slit, so we use an average slit width of $1^{\prime \prime} .25$. If we consider neighbors within $20 \mathrm{kpc}\left(\sim 3^{\prime \prime}\right)$, the fraction of area within that radius of the quasar subtended by the slit is $\sim 13 \%$ (ignoring slit losses for miscentered objects). Thus we would have detected 1 in $\sim 7.5$ galaxy neighbors in our quasar survey. Experimenting with a single exposure of J0256+0019, if the Ly $\alpha$ emission were a factor of $\sim 2$ fainter, it would have still been noticeable during the data reduction. From this crude analysis we conclude that less than 1 in $\sim 55 / 7.5 \approx 7$ quasars in our $z=5$ survey have a companion with a Ly $\alpha$ flux within a factor of two of the J0256+0019 companion.

It is somewhat easier to draw statistical conclusions from the J0050+3445 observations. A total of $29 z \sim 6$ quasars were observed during the HST SNAP survey. Brighter quasars $\left(z_{\mathrm{AB}}<20.5\right)$ had a total exposure time of $300 \mathrm{~s}$, while the fainter objects were exposed for $1200 \mathrm{~s}$. J0050+3445 was in the bright sample and its companion galaxy was detected near the limit of the imaging; the faint sample could reach galaxies $\sim 1.6$ times fainter. Thus we could have detected companion galaxies to a limit of $\approx 5 L^{*}$ for the 23 bright objects observed, and $\approx 2 L^{*}$ for the 6 faint objects. One other bright quasar has excess emission in the SNAP imaging and is scheduled for further observations in Cycle 21; as part of the search for gravitational lenses we can rule out any other companions at separations $<3$ ". Thus the incidence of UV-bright galaxies associated with quasars at $z \sim 6$ is $\lesssim 2 / 29$ for $\gtrsim 5 L^{*}$ galaxies and $<1 / 6$ for $2 \lesssim L \lesssim 5 L^{*}$ galaxies.

At least within the context of our observations, bright companions to high-redshift quasars are uncommon. We conclude with some final thoughts related to the incidence of such systems.

\section{CONCLUSIONS}

We have identified two galaxies in close proximity to highredshift quasars. The first object is located 1".8 from a $z=$ 4.9 quasar (SDSS J025617.7+001904) and was discovered serendipitously based on excess $\operatorname{Ly} \alpha$ emission present in the spectroscopic slit during the course of an MMT survey of $z \sim 5$ 
quasars. Imaging and moderate depth optical spectroscopy with the LBT confirms that the galaxy is bright $(i=23.6)$ and has strong Ly $\alpha$ emission at the quasar redshift as well as UV continuum emission. The second companion galaxy is $<1^{\prime \prime}$ from a highly luminous $z=6.25$ quasar (CFHQS J005006.6+344522). HST imaging demonstrates that it is a relatively bright $(Y=25)$ optical dropout and highly likely to be at the same redshift as the quasar.

Both galaxies are among the most luminous galaxies known at high redshift, with $M_{1500}=-22.7$ for the J0256+0019 companion and $M_{1500}=-21.8$ for the J0050+3445 companion. We have considered possible sources for their emission, including internal AGNs or fluorescence from the nearby quasars, and for both objects conclude that the observed emission is likely dominated by star formation activity within the galaxies. The coincidence of highly star-forming galaxies near luminous quasars is broadly consistent with the scenario where high-redshift quasars are fueled by major mergers.

This study is not the first to find close companion galaxies to high-redshift quasars. The first galaxy to have a reported spectroscopic redshift $z>2$ was a companion to the $z=3.2$ quasar QSO PKS 1614+051 with a separation of 7" (Djorgovski et al. 1985). This galaxy was selected from narrow-band Ly $\alpha$ imaging of the quasar, and was the only instance of a companion out of five objects surveyed. The $z=4.7$ quasar BR 12020725 was found to have multiple companions within $\sim 5^{\prime \prime}$, two discovered via Ly $\alpha$ (Hu et al. 1996; Petitjean et al. 1996) and one in the submillimeter (Omont et al. 1996). While searching for $\mathrm{CO}(2-1)$ line emission from the host galaxies of five quasars at $z \sim 6$, Wang et al. (2011) found one clear detection of a companion galaxy $1^{\prime \prime}$.2 from a $z=6.18$ quasar, and a marginal $\sim 2 \sigma$ detection of extended $\mathrm{CO}$ emission $\sim 0$ '. 8 from a $z=5.85$ quasar. These studies either targeted quasar fields under the expectation they would point to a local galaxy overdensity, or targeted quasar hosts while being sensitive to any nearby companions.

One can then ask why companion galaxies are not observed more frequently, given the observational attention paid to highredshift quasars and the hypothesis that major mergers play a key role in triggering high-redshift quasars. One explanation is that the merging timescales are brief, so that observing quasar-galaxy pairs would be rare. Indeed, the "blowout" phase that leads to an unobscured quasar may occur only after the progenitor galaxies have fully coalesced (e.g., Hopkins et al. 2006). In the scenario where successive galactic encounters fuel the growth of high-redshift quasars, the duty cycles of unobscured activity for both star formation (in the progenitor galaxies) and $\mathrm{BH}$ growth may be sufficiently short (relative to the quasar lifetime) that the probability of observing the two simultaneously at restframe UV/optical wavelengths is low. Larger, more sensitive surveys at FIR wavelengths with ALMA would better address this question. At rest-UV wavelengths, Jiang et al. (2013) found that $\sim 50 \%$ of bright $z \sim 6$ galaxies show evidence for recent mergers or interactions in HST observations. However, observing such faint features in luminous quasar host galaxies would be exceedingly difficult.

A final speculation motivated by our observations is that the proximity of a UV-bright galaxy to a rapidly growing SMBH at high redshift may be indicative of a form of positive feedback. Dijkstra et al. (2008) outline a model where massive seed BHs for $z \sim 6$ quasars form at even higher redshifts $(z \sim 10)$ from close pairs $(\lesssim 10 \mathrm{kpc})$ of dark matter halos. In this model, one halo hosts a bright star-forming galaxy with a strong Lyman-Werner band flux that photo-dissociates $\mathrm{H}_{2}$ molecules in the neighboring halo, preventing it from cooling below the atomic cooling threshold. The result is direct collapse of the neighboring halo gas into a massive $\left(\sim 10^{4-6} M_{\odot}\right) \mathrm{BH}$, providing a seed mechanism for SMBHs. However, the original ionizing source at $z \sim 10$ would likely coalesce with the quasar host by $z \sim 6$, and not explain our observations in which a bright companion galaxy is contemporaneous with an already $\gtrsim 10^{8} M_{\odot}$ BH. On the other hand, observing a companion galaxy to a quasar well after the initial formation of the $\mathrm{BH}$ is consistent with the picture where multiple mergers are needed to grow high-redshift quasars (e.g., Li et al. 2007), drawing at least some connection between the model of Dijkstra et al. (2008) and our observations.

The authors thank George Becker for providing the ESI spectrum of J0050+3445. I.D.M. thanks Desika Narayanan and Dan Stark for helpful discussions.

Support for programs \#12184 and \#12493 was provided by NASA through a grant from the Space Telescope Science Institute, which is operated by the Association of Universities for Research in Astronomy, Inc., under NASA contract NAS 5-26555. I.D.M. and X.F. acknowledge additional support from NSF grants AST 08-06861 and AST 11-07682.

The LBT is an international collaboration among institutions in the United States, Italy, and Germany. LBT Corporation partners are: The University of Arizona on behalf of the Arizona University System; Istituto Nazionale di Astrofisica, Italy; LBT Beteiligungsgesellschaft, Germany, representing the Max-Planck Society, the Astrophysical Institute Potsdam, and Heidelberg University; The Ohio State University, and The Research Corporation, on behalf of The University of Notre Dame, University of Minnesota, and University of Virginia. This paper used data obtained with the MODS spectrographs built with funding from NSF grant AST-9987045 and the NSF Telescope System Instrumentation Program (TSIP), with additional funds from the Ohio Board of Regents and the Ohio State University Office of Research.

The MMT Observatory is a joint facility of the University of Arizona and the Smithsonian Institution.

Facilities: MMT (Red Channel spectrograph, SWIRC), LBT (MODS), HST (ACS,WFC3), Sloan

\section{REFERENCES}

Ahn, C. P., Alexandroff, R., Allende Prieto, C., et al. 2012, ApJS, 203, 21

Alexandroff, R., Strauss, M. A., Greene, J. E., et al. 2013, MNRAS, 435, 3306 Bahcall, J. N., Kirhakos, S., Saxe, D. H., \& Schneider, D. P. 1997, ApJ, 479,642

Becker, G. D., Sargent, W. L. W., Rauch, M., \& Calverley, A. P. 2011, ApJ, 735,93

Benítez, E., Krongold, Y., González, J. J., et al. 2013, Rev. Mex. Astron. Astrofis. Ser. Conf., 42, 62

Bennert, N., Canalizo, G., Jungwiert, B., et al. 2008, ApJ, 677, 846

Bertin, E., \& Arnouts, S. 1996, AJ, 117, 393

Bian, F., Fan, X., Jiang, L., et al. 2012, ApJ, 757, 139

Bouwens, R. J., Illingworth, G. D., Oesch, P. A., et al. 2011, ApJ, 737, 90 Bouwens, R. J., Illingworth, G. D., Oesch, P. A., et al. 2014, arXiv:1403.4295 Bowler, R. A. A., Dunlop, J. S., McLure, R. J., et al. 2012, MNRAS, 426, 2772 Bowler, R. A. A., Dunlop, J. S., McLure, R. J., et al. 2014, MNRAS, 440, 2810 Brown, W. R., McLeod, B. A., Geary, J. C., \& Bowsher, E. C. 2008, Proc. SPIE, 7014, 90

Carilli, C. L., Riechers, D., Walter, F., et al. 2013, ApJ, 763, 120

Comerford, J. M., Haiman, Z., \& Schaye, J. 2002, ApJ, 580, 63

Curtis-Lake, E., McLure, R. J., Dunlop, J. S., et al. 2013, MNRAS, 429, 302

Dijkstra, M., Haiman, Z., Mesinger, A., \& Wyithe, J. S. B. 2008, MNRAS, 391, 1961 
Di Matteo, T., Colberg, J., Springel, V., Hernquist, L., \& Sijacki, D. 2008, AJ, 676,33

Djorgovski, S., Spinrad, H., McCarthy, P., \& Strauss, M. A. 1985, ApJL, 299, L1

Fan, X., Narayanan, V. K., Lupton, R. H., et al. 2001, AJ, 122, 2833

Fanidakis, N., Maccio, A. V., Baugh, C. M., Lacey, C. G., \& Frenk, C. S. 2013, MNRAS, 436, 315

Filippenko, A. V. 1985, AJ, 90, 1172

Fukugita, M., Ichikawa, T., Gunn, J. E., et al. 1996, AJ, 111, 1748

Giavalisco, M., Ferguson, H. C., Koekemoer, A. M., et al. 2004, ApJL, 600, L93

Gould, A., \& Weinberg, D. H. 1996, ApJ, 468, 462

Grogin, N. A., Kocevski, D. D., Faber, S. M., et al. 2011, ApJS, 197, 35

Gunn, J. E. 1971, ApJL, 164, L113

Guo, Y., Ferguson, H. C., Giavalisco, M., et al. 2013, ApJS, 207, 24

Haiman, Z. 2013, in The First Galaxies, ed. T. Wiklind, B. Mobasher, \& V. Bromm (Astrophysics and Space Science Library, Vol. 396; Berlin: Springer), 293

Haiman, Z., \& Rees, M. J. 2001, ApJ, 556, 87

Hernquist, L. 1989, Natur, 340, 687

Hopkins, P. F., Hernquist, L., Cox, T. J., \& Keres, D. 2008, ApJS, 175, 356

Hopkins, P. F., Hernquist, L., Cox, T. J., et al. 2006, ApJS, 163, 1

Horne, K. 1986, PASP, 98, 609

Hu, E. M., McMahon, R. G., \& Egami, E. 1996, ApJL, 459, L53

Husband, K., Bremer, M. N., Stanway, E. R., et al. 2013, MNRAS, 432, 2869

Hynes, R. I. 2002, A\&A, 382, 752

Isaak, K. G., McMahon, R. G., Hills, R. E., \& Withington, S. 1994, MNRAS, 269, L28

Jiang, L., Egami, E., Fan, X., et al. 2013, ApJ, 773, 153

Jiang, L., Fan, X., Bian, F., et al. 2009, AJ, 138, 305

Jiang, L., Fan, X., Bian, F., et al. 2014, ApJS, 213, 12

Juneau, S., Dickinson, M., Bournaud, F., et al. 2013, ApJ, 764, 176

Kashikawa, N., Kitayama, T., Doi, M., et al. 2007, ApJ, 663, 765

Kashikawa, N., Shimasaku, K., Matsuda, Y., et al. 2011, ApJ, 734, 119

Kauffmann, G., \& Haehnelt, M. 2000, MNRAS, 311, 576

Keel, W. C., Lintott, C. J., Schawinski, K., et al. 2012, AJ, 144, 66

Keeton, C. R., Kuhlen, M., \& Haiman, Z. 2005, ApJ, 621, 559

Kelson, D. D. 2003, PASP, 115, 688

Kennicutt, R. C. J. 1998, ARA\&A, 36, 189

Kim, S., Stiavelli, M., Trenti, M., et al. 2009, ApJ, 695, 809

Koekemoer, A. M., Faber, S. M., Ferguson, H. C., et al. 2011, ApJS, 197, 36

Komatsu, E., Smith, K. M., Dunkley, J., et al. 2011, ApJS, 192, 18

Lawrence, A., Warren, S. J., Almaini, O., et al. 2007, MNRAS, 379, 1599
Leggett, S. K., Currie, M. J., Varricatt, W. P., et al. 2006, MNRAS, 373, 781

Li, Y., Hernquist, L., Robertson, B., et al. 2007, ApJ, 665, 187

McGreer, I. D., Jiang, L., Fan, X., et al. 2013, ApJ, 768, 105

McLure, R. J., Dunlop, J. S., Bowler, R. A. A., et al. 2013, MNRAS, 432, 2696

Mechtley, M., Windhorst, R. A., Ryan, R. E., et al. 2012, ApJL, 756, L38

Mortlock, D. J., Warren, S. J., Venemans, B. P., et al. 2011, Natur, 474, 616

Ohyama, Y., Taniguchi, Y., \& Shioya, Y. 2004, AJ, 128, 2704

Oke, J. B., \& Gunn, J. E. 1983, AJ, 266, 713

Omont, A., Petitjean, P., Guilloteau, S., et al. 1996, Natur, 382, 428

Ouchi, M., Ono, Y., Egami, E., et al. 2009, ApJ, 696, 1164

Petitjean, P., Pécontal, E., Valls-Gabaud, D., \& Chariot, S. 1996, Natur, 380,411

Pogge, R. W., Atwood, B., Belville, S. R., et al. 2006, Proc. SPIE, 6269, 16

Richards, G. T., Kruczek, N. E., Gallagher, S. C., et al. 2011, AJ, 141, 167

Richards, G. T., Strauss, M. A., Pindor, B., et al. 2004, AJ, 127, 1305

Schenker, M. A., Robertson, B. E., Ellis, R. S., et al. 2013, ApJ, 768, 196

Schlegel, D. J., Finkbeiner, D. P., \& Davis, M. 1998, AJ, 500, 525

Shapley, A. E., Steidel, C. C., Pettini, M., \& Adelberger, K. L. 2003, ApJ, 588, 65

Shen, Y., Richards, G. T., Strauss, M. A., et al. 2011, ApJS, 194, 45

Shull, J. M., Stevans, M., \& Danforth, C. W. 2012, ApJ, 752, 162

Stark, D. P., Ellis, R. S., Bunker, A., et al. 2009, ApJ, 697, 1493

Stiavelli, M., Djorgovski, S. G., Pavlovsky, C., et al. 2005, ApJL, 622, L1

Tanaka, T., \& Haiman, Z. 2009, ApJ, 696, 1798

Turner, E. L., Ostriker, J. P., \& Gott, J. R. 1984, ApJ, 284, 1

Urrutia, T., Lacy, M., \& Becker, R. H. 2008, ApJ, 674, 80

Utsumi, Y., Goto, T., Kashikawa, N., et al. 2010, ApJ, 721, 1680

Vanden Berk, D. E., Richards, G. T., Bauer, A., et al. 2001, AJ, 122, 549

Venemans, B. P., Findlay, J. R., Sutherland, W. J., et al. 2013, ApJ, 779,24

Vestergaard, M., \& Peterson, B. M. 2006, ApJ, 641, 689

Volonteri, M. 2010, ARA\&A, 18, 279

Walter, F., Bertoldi, F., Carilli, C., et al. 2003, Natur, 424, 406

Wang, R., Carilli, C. L., Neri, R., et al. 2010, ApJ, 714, 699

Wang, R., Wagg, J., Carilli, C. L., et al. 2011, ApJL, 739, L34

Wang, R., Wagg, J., Carilli, C. L., et al. 2013, ApJ, 773, 44

Williams, R. J., Wagg, J., Maiolino, R., et al. 2014, MNRAS, 439, 2096

Willott, C. J., Albert, L., Arzoumanian, D., et al. 2010a, AJ, 140, 546

Willott, C. J., Delorme, P., Reylé, C., et al. 2010b, AJ, 139, 906

Willott, C. J., Percival, W. J., McLure, R. J., et al. 2005, ApJ, 626, 657

York, D. G., Adelman, J., Anderson, J. E., Jr., et al. 2000, AJ, 120, 1579

Zheng, W., Overzier, R. A., Bouwens, R. J., et al. 2006, ApJ, 640, 574 